\title{
INSERÇÃO DO BIM NA FORMAÇÃO EM ENGENHARIA CIVIL: ANÁLISE E REFLEXÕES
}

\author{
Aparecida Hippert ${ }^{(1)}$, Mauricio Aguilar Molina ${ }^{(1)}$ \\ (1) Universidade Federal de Juiz de Fora, Juiz de Fora - MG
}

\begin{abstract}
Resumo
A adoção do BIM - Building Information Modeling - constitui um novo paradigma para o desenvolvimento de empreendimentos no contexto da chamada Indústria 4.0. Embora muitas empresas ainda encontrem dificuldades para a adoção do BIM em suas rotinas de trabalho, este é um caminho sem volta. O BIM pode cobrir todas as fases do ciclo de vida de um empreendimento e isso exige novas competências dos profissionais do setor de AECO. Neste sentido, este trabalho tem por objetivo fazer uma análise sobre as formas de inserção do BIM na formação em Engenharia Civil, de maneira a atender às demandas atuais do mercado em termos de inovação tecnológica. Para isso é feito um levantamento bibliográfico de algumas experiências sobre o ensino do BIM no Brasil e no exterior. Como exemplo, é feito um estudo de caso tendo como base o Curso de Engenharia Civil da Universidade Federal de Juiz de Fora. Os resultados obtidos são analisados e discutidos à luz de relatos recentes de introdução do BIM encontrados na literatura, ao tempo em que se tenta identificar as dificuldades e os desdobramentos necessários para uma adoção mais efetiva do BIM na formação de profissionais da Engenharia Civil.
\end{abstract}

\section{Introdução}

A construção civil passa por mudanças significativas com a adoção de um novo paradigma para o desenvolvimento de empreendimentos de engenharia e arquitetura, o BIM. Ele envolve um conjunto de tecnologias de projeto e processos produtivos do setor da AECO de maneira a permitir a geração e gestão de dados de construção ao longo do ciclo de vida de um empreendimento, envolvendo a coordenação entre as diferentes disciplinas do projeto.

No Brasil, o número de empresas que utilizam o BIM vem crescendo com o surgimento de exigências de desenvolvimento de projetos em BIM pelas instâncias governamentais. Atualmente, a instituição da Estratégia Nacional para disseminação do BIM [1] pelo governo federal vem reforçar a demanda pela sua inserção nos currículos dos cursos de engenharia civil. Porém, a incorporação do BIM nos currículos destes cursos não é tarefa simples [2]. São muitas as dificuldades a serem enfrentadas pelos interessados em realizar esta empreitada visto que uma série de mudanças precisa ser realizada. 
Este artigo tem por objetivo apresentar e discutir a inclusão do BIM no curso de Engenharia Civil da Universidade Federal Juiz de Fora (UFJF) através da experiencia da criação de uma disciplina eletiva sobre o assunto e sua implementação nos anos recentes. Os resultados obtidos a partir de um questionário respondido pelos alunos no último semestre, contrastados com as observações dos docentes responsáveis pela disciplina, são analisados e discutidos à luz de relatos recentes de introdução do BIM no currículo encontrados na literatura, identificando as dificuldades vivenciadas e as alternativas para se alcançar uma adoção mais efetiva do BIM no ensino no curso de Engenharia Civil da UFJF.

\section{Fundamentação}

\subsection{O BIM}

O BIM é "uma tecnologia de modelagem e um grupo associado de processos para produção, comunicação e análise do modelo de construção" [3]. Desse modo, todos os agentes envolvidos no ciclo de vida da edificação trabalham de forma integrada sobre um modelo único do edifício, possibilitando a integração dos processos de projeto e construção, o qual resulta em obras de melhor qualidade, a custos mais baixos e prazo de execução do projeto reduzida [4]. “... A utilização de uma prática baseada no BIM pode ter um papel decisivo na melhoria das fases do projeto, auxiliando na geração de propostas coerentes com as solicitações dos clientes, na integração dos projetos, entre si e com a construção, na redução do tempo e do custo da construção" [5].

Modelagem paramétrica e interoperabilidade constituem os pilares básicos do BIM [3]. A incorporação de informação não geométrica possibilita a definição de parâmetros de projeto e regras associadas à geometria. Dessa forma, um modelo é organizado em um complexo banco de dados, do qual é possível extrair relatórios e informações para checagem de inconsistências entre objetos e, também, permite a incorporação de conhecimentos de projeto no modelo. A interoperabilidade tem a ver com a derrubada das barreiras entre softwares de diferentes fabricantes, a partir do qual eles podem estabelecer uma interação através de uma linguagem comum e aberta. Ela é essencial para a modelagem paramétrica, pois envolve a organização de informação de natureza e origens diversos para serem acessíveis aos diferentes softwares BIM, em diferentes etapas no ciclo de vida da edificação [3].

O desenvolvimento do BIM trilhou vários caminhos. Por um lado, a evolução das Tecnologias da Informação e da Comunicação (TIC), com o aumento da capacidade de processamento gráfico e de dados dos equipamentos utilizados para modelagem. Por outro, o desenvolvimento das redes de computadores até a consolidação da Internet criou um contexto no qual velhas práticas de projeto perderam seu sentido e deram lugar a espaços inéditos para o resgate de uma componente importante no projeto: a colaboração, o que implica na necessidade de reformulação dos processos da cadeia de valor do setor.

O BIM tem impacto em todas as etapas do ciclo de vida da edificação, pois todas as informações são estruturadas de maneira integrada e consistente [3]. Isso faz possível a realização de diferentes análises na fase de projeto a partir da representação da geometria do edifício, que incorpora também informações de caráter não geométrico, com impacto na qualidade dos dados obtidos e seu efeito na coordenação entre as disciplinas do projeto e a redução de tempo e custos. 
A fase de planejamento adquire uma nova dimensão a partir da superação da visão tradicional do gráfico de Gantt pela modelagem 4D, que possibilita uma análise da programação através da visualização não apenas de eventuais conflitos temporais, mas também espaciais, não evidenciados no modelo de Gantt. Na fase de construção, mesmo com uso de ferramentas CAD, os desenhos bidimensionais constituem um grande empecilho e dão origem a grande parte dos problemas enfrentados nesta etapa, não apenas em termos de prazos e orçamento, mas principalmente de compatibilidade entre disciplinas do projeto.

Com o BIM é possível a obtenção de estimativas de quantitativos com alto grau de confiabilidade, de modo que o empreiteiro tem condições de definir com precisão quantidades de materiais de construção, as quais - num contexto tradicional de trabalho - apresentam os maiores desvios em relação com estimativas preliminares, com impactos potenciais no orçamento. O BIM tem grande impacto na fase de operação e manutenção do edifício, pois fornece um repositório completo de informações detalhadas sobre todos os componentes do sistema, as quais podem ser usadas para gestão da manutenção [3]. Ao mesmo tempo em que o gerente tem acesso fácil e rápido às informações essenciais de manutenção, ele tem a possibilidade de atualizar tais informações no modelo virtual do edifício em função das atualizações feitas na estrutura física. Assim, se qualquer elemento apresentar problemas, o fornecedor desse elemento poderá ser prontamente identificado e contatado sua substituição.

\subsection{Ensino de BIM}

A incorporação do BIM no currículo acadêmico de cursos de Arquitetura e Engenharia Civil não é simples. Em uma revisão bibliográfica, foram constatadas várias dificuldades nesse sentido, tais como: falta de métodos de ensino e materiais didáticos adequados e específicos para o BIM, falta de qualificação do quadro docente tradicional face à rápida evolução das novas tecnologias, necessidade de forte investimento em recursos de hardware e software, além de competências em TIC comumente não desenvolvidas pelos discentes ao longo do curso [2]. Por fim, o currículo do curso não dispõe de espaço para novas disciplinas. Tais dificuldades podem ser sintetizadas em três aspectos [6]: a dificuldade no aprendizado de softwares BIM pelos discentes, principalmente pela falta de tempo nos últimos semestres do curso, a incompreensão dos novos processos do BIM e, por último, questões relacionadas às circunstâncias do ambiente acadêmico.

A despeito das dificuldades apontadas, algumas universidades internacionais vêm trabalhando na implantação do BIM na grade curricular de seus cursos. Esse movimento começou em 2003 quando várias escolas começaram a ensinar ferramentas BIM, o que se intensificou entre 2006 e 2009 [7], no intuito de atender a uma demanda do mercado de trabalho que procurava por profissionais habilitados a trabalhar em projetos BIM $[4,7,8]$.

No Brasil, a inserção do BIM nos currículos é ainda pontual e discreta [9], sendo variadas as dificuldades encontradas [10]. Para estes autores, a necessidade de integração de diferentes conteúdos e disciplinas, além da colaboração entre professores, são duas das maiores dificuldades a serem vencidas para a inclusão do BIM nos processos de ensino-aprendizagem. Além destas podem ser citadas: "a necessidade de máquinas e programas sofisticados e atualizados; falta de espaço na matriz curricular dos cursos de graduação para inserir um tema tão vasto e complexo como o BIM; o custo associado à rápida obsolescência da tecnologia, dentre outros" [11]. Estas barreiras podem ser classificadas em relação a pessoas, metodologias didáticas, regulamentação, infraestrutura e tecnologia e por último financeira. 
Foram identificadas àquela época, em 2013, instituições como a Universidade Federal de Alagoas, Universidade Federal de São Carlos, Universidade Presbiteriana Mackenzie, Centro Universitário Barão de Mauá (SP) e Universidade Estadual de Campinas que já vinham experimentando a implementação do ensino de BIM em seus cursos de arquitetura e engenharia civil [9]. Atualmente, o ensino de BIM tem crescido conforme pôde ser visto no II Encontro Nacional sobre o Ensino de BIM [12], onde foram apresentados relatos de experiências didáticas bem-sucedidas em relação com a criação de disciplinas e grupos de estudo BIM, inserção de conteúdo BIM em disciplinas existentes, integração de disciplinas, dentre outros.

\subsection{Ensino de BIM em cursos de Engenharia Civil}

As experiências de utilização do BIM segundo os estágios de implementação e os níveis de competência são consignadas na Tabela 1 [9].

Tabela 1: Parâmetros de classificação das experiências didáticas de ensino de BIM

\begin{tabular}{|c|c|c|c|c|c|}
\hline \multirow[b]{2}{*}{$\begin{array}{l}\text { Nível de } \\
\text { competência }\end{array}$} & \multirow[b]{2}{*}{$\begin{array}{c}\text { Classificação } \\
\text { das } \\
\text { Habilidades }\end{array}$} & \multirow{2}{*}{$\begin{array}{c}\text { Estágio } \\
\text { de } \\
\text { adoção } \\
\text { de BIM }\end{array}$} & \multicolumn{3}{|c|}{ Parâmetros de classificação } \\
\hline & & & $\begin{array}{l}\text { Modelo de } \\
\text { informação }\end{array}$ & $\begin{array}{c}\text { Fases do } \\
\text { ciclo de vida }\end{array}$ & $\begin{array}{c}\text { Produtos gerados } \\
\text { na experiência } \\
\text { didática }\end{array}$ \\
\hline Introdutório & $\begin{array}{l}\text { Habilita } \\
\text { modelador }\end{array}$ & Primeiro & $\begin{array}{l}\text { Modelagem e } \\
\text { produtividade }\end{array}$ & Uma fase & $\begin{array}{l}\text { Modelagem } \\
\text { paramétrica, } \\
\text { quantitativos. } \\
\text { documentação }\end{array}$ \\
\hline Intermediário & $\begin{array}{l}\text { Habilita } \\
\text { analista }\end{array}$ & Segundo & $\begin{array}{l}\text { Integração de } \\
\text { modelos com uso } \\
\text { aplicado dos } \\
\text { modelos de } \\
\text { informação }\end{array}$ & Duas fases & $\begin{array}{c}\text { Simulações } \\
\text { (dimensionamento, } \\
\text { ambientais, 4D, } \\
\text { 5D...), } \\
\text { compatibilização c } \\
\text { planejamento } \\
\text { (caminhos críticos, } \\
\text { linha de balanço) }\end{array}$ \\
\hline Avançado & $\begin{array}{l}\text { Habilita } \\
\text { gerente }\end{array}$ & Terceiro & $\begin{array}{l}\text { Desenvolvimento } \\
\text { compartilhado } \\
\text { holístico do } \\
\text { modelo de } \\
\text { informação }\end{array}$ & Três fases & $\begin{array}{l}\text { Introdução a IPD. } \\
\text { Colaboração } \\
\text { envolvendo } \\
\text { múltiplos agentes. } \\
\text { Criação } \\
\text { compartilhada }\end{array}$ \\
\hline
\end{tabular}

No nível introdutório, a atenção está na modelagem paramétrica aplicada a uma fase do ciclo de vida (projeto, construção ou operação). No nível intermediário o foco se volta para o compartilhamento multidisciplinar do modelo em até duas disciplinas e demanda uma coordenação de projetos. No último estágio - avançado - a ênfase passa a ser no desenvolvimento compartilhado e colaborativo do modelo, com a consideração de todas as fases do empreendimento e as múltiplas disciplinas da área de AECO. 
Nos casos analisados estes autores verificaram que o processo de implantação do BIM no ensino abrange os níveis de competência introdutório e intermediário e acontecem de forma gradual [9]. Além disto, a maioria das experiências considera o ensino de BIM em disciplinas isoladas, tendo algumas exceções em iniciativas de integração entre as disciplinas de projeto arquitetônico e estrutural. Não foram encontrados relatos que caracterizassem o nível de competência avançado. Tampouco foram encontradas experiências que, mesmo trabalhando com modelos compartilhados e integrados abordassem todas as fases do empreendimento, caracterizando o nível avançado de adoção do BIM.

As abordagens adotadas comumente para ensino do BIM são [7]: utilizá-lo em uma ou duas disciplinas, ou em várias disciplinas do curso. A primeira abordagem considera o ensino do BIM em uma disciplina do início do curso e outra no final. Na segunda abordagem a modelagem BIM é utilizada para ajudar o aluno a entender determinado conteúdo. Desta forma, o BIM vem sendo introduzido em várias áreas do currículo como, por exemplo, representação gráfica digital, disciplina BIM, Trabalho de Conclusão de Curso (TCC), dentre outros. No caso da disciplina BIM, ela pode ser eletiva ou integrar o currículo [7].

Segundo Barison e Santos [13] a melhor forma de inserir BIM no currículo deveria ser através de uma ou mais disciplinas; no entanto, como muitos professores não possuem conhecimento ou experiência para tal mudança os autores desenvolveram uma ferramenta para auxiliá-los nesta tarefa. Por outro lado, é difícil a criação de mais do que duas disciplinas sobre BIM no curso, o que acaba por levar a abordagens apenas introdutórias do tema [10]. Além disso, a adoção integrada do BIM no currículo apresenta como maior desvantagem a necessidade de uma maior quantidade de professores que compreendam o BIM e sejam capazes de desenvolver o trabalho de forma integrada. Esta estratégia, porém, traz a vantagem de permitir aos alunos trabalharem no BIM em diferentes enfoques e aplicações, possibilitando uma aprendizagem mais robusta. Para ajudar os professores nesta tarefa, foi desenvolvido um método para identificar interfaces entre o currículo do engenheiro civil e BIM [10]. Para um ensino sólido do BIM os autores recomendam começar nas disciplinas do núcleo básico, no primeiro ano do curso, com uma nova estruturação para as disciplinas de expressão gráfica, que passariam a considerar o ensino de BIM [10]. É recomendável também que o BIM seja introduzido no currículo do curso a partir dos seus fundamentos [4], para que os futuros profissionais estejam capacitados a atuar no setor de AECO, onde o meio de expressão gráfica e comunicação de projetos está centrado nos modelos 3D e não mais nos modelos CAD 2D.

A partir de um levantamento junto aos alunos e professores do curso de Engenharia Civil da UFES é proposta uma série de recomendações para a implementação de novas metodologias de ensino-aprendizagem no curso de engenharia civil daquela instituição [14]. Porém, é preciso ter em consideração que, em função das diferenças entre instituições, não é viável a definição de um modelo único a ser adotado por todas as instituições, de modo tal que a inserção do BIM deve ser feita de acordo com o projeto pedagógico de cada curso.

\section{O ensino de BIM no curso de Engenharia Civil da UFJF}

\subsection{O curso e suas disciplinas}

O curso de Engenharia Civil da UFJF está organizado em sete áreas de conhecimento: Materiais de Construção Civil, Planejamento e Construção, Estruturas, Recursos Hídricos e Saneamento, Mecânica dos Sólidos, Geotecnia e Transportes e Levantamentos, distribuídas pelos cinco 
departamentos da Faculdade de Engenharia: Construção Civil, Estruturas, Engenharia Sanitária e Ambiental, Mecânica Aplicada e Computacional, e Transportes e Geotecnia. A estrutura curricular do curso está organizada segundo 3 grupos de conteúdo obrigatórios [15]: Conteúdos Básicos, Conteúdos Profissionalizantes e Conteúdos Específicos. A integralização da carga horária total do curso requer cursar todas as disciplinas obrigatórias, além de 18 créditos eletivos (um crédito corresponde a uma hora-aula/semana ao longo do semestre), além do desenvolvimento de um TCC e o estágio curricular obrigatório.

Os alunos são incentivados também a participar de atividades complementares. com o objetivo de se inteirarem acerca das diferentes áreas de conhecimento, estabelecendo parâmetros práticos para a atuação profissional, tornando-se uma ponte entre a teoria e a prática. Apesar disso, a formação dos engenheiros civis da UFJF ainda é pautada por metodologias e técnicas tradicionais de corte disciplinar, o que deixa pouco espaço para a criatividade, a inovação e a visão holística do projeto [16].

No caso das disciplinas do Núcleo Básico da área da Expressão Gráfica (Desenho Técnico, Geometria Descritiva, Desenho Arquitetônico e Desenho Auxiliado por Computador), observase que, embora seus programas apresentem conteúdo de desenho geométrico e CAD 2D, além de noções básicas de projeto arquitetônico, não se percebe uma conexão explícita entre as competências básicas que deveriam desenvolvidas nesta área [17] e as competências de projeto que deveriam ser desenvolvidas em disciplinas profissionalizantes e específicas. De fato, não é possível encontrar no currículo atual do curso disciplinas ou atividades que desenvolvam o projeto de Engenharia Civil na sua plenitude e diversidade [16].

\subsection{A disciplina eletiva BIM}

Em 2012 foi criada no Departamento de Construção Civil a disciplina eletiva BIM, com dois créditos, com o objetivo de iniciar sua introdução na formação dos Engenheiros Civis da UFJF. A disciplina parte da premissa de que o BIM não é apenas um conjunto de ferramentas de software para modelagem 3D, mas um novo paradigma no setor da AECO, o qual promove uma simbiose entre diferentes competências, as quais comumente são desenvolvidas de modo estanque no currículo do curso. A ementa da disciplina é aberta e contempla como tópicos: a indústria da Construção Civil, o conceito de BIM, e aplicações de softwares BIM na modelagem de projetos. A disciplina tem como objetivos: discutir o conceito de BIM a partir de uma perspectiva sistêmica da Construção Civil e conhecer algumas ferramentas de software para modelagem 3D e 4D. São oferecidas 20 vagas por semestre.

\subsection{Metodologia de trabalho da disciplina}

O desenvolvimento da disciplina envolve uma discussão teórico em torno de conceitos relacionados com a evolução da construção civil a partir dos avanços em matéria de métodos e novas tecnologias construtivas. São apresentados alguns textos selecionados para serem discutidos pela turma no ambiente virtual da disciplina.

A parte prática consiste em uma atividade de modelagem em equipes de três a quatro discentes, as quais desenvolvem um modelo 3D de um projeto simples 3D, incluindo uma modelagem básica da estrutura e do MEP (Mechanical, Electrical \& Plumbing), além de um modelo 4D a partir de um cronograma básico. Para esse fim são usados softwares com licenciamento educacional.

A fim de explorar a dimensão colaborativa, cada equipe é incumbida do desenvolvimento e modelagem de um projeto de arquitetura, com softwares Autodesk Revit e Graphisoft Archicad. 
$\mathrm{Na}$ etapa seguinte, cada equipe "terceiriza" o desenvolvimento de projetos complementares (i.e., estrutura e MEP) para as outras equipes, fornecendo, para esse fim, o projeto arquitetônico desenvolvido no início. A modelagem é feita com o software Autodesk Revit e as análises básicas com os softwares Autodesk Robot ou TQS.

Em função das limitações de tempo de uma disciplina eletiva de dois créditos, o aprendizado dos softwares fica sob a responsabilidade pessoal dos discentes, com uso do farto material disponível na Internet e recursos desenvolvidos para esse propósito no contexto da disciplina. Assim, cada equipe assume o compromisso de desenvolver seu projeto e os projetos "contratados" pelas outras equipes dentro dos prazos previstos no planejamento da disciplina. A compatibilização dos projetos é feita com os softwares Autodesk Navisworks Manage, Trimble Tekla BIMsight (desktop) e usBIM (web), o que possibilita a interação entre as equipes na coordenação das diferentes disciplinas previstas no exercício e a compreensão do conceito de Interoperabilidade.

\subsection{Avaliação dos discentes}

De maneira a verificar a percepção dos discentes quanto ao aprendizado de BIM na disciplina eletiva, no segundo semestre letivo de 2019 foi aplicado um questionário com as questões: "Quais foram as principais dificuldades encontradas na disciplina? Elas foram resolvidas? Como?", "Você considera que o conhecimento sobre BIM deveria ser obrigatório para todos os formandos do curso de Engenharia Civil?", "Na sua opinião, o BIM deveria ser abordado em apenas uma ou duas disciplinas ou permear o curso inteiro?". O questionário foi enviado para os 19 alunos inscritos, dos quais apenas 13 responderam. Os alunos inscritos estão na faixa entre 20 e 22 anos de idade.

\section{Resultados e discussão}

Os resultados mostraram que a motivação básica dos alunos foi conhecer os softwares de modelagem BIM 3D apresentados na disciplina. Eles também manifestaram interesse na coordenação de projetos e gerenciamento do ciclo de vida do projeto. Em geral, houve consenso de que a disciplina possibilitou obter uma maior percepção da complexidade do ciclo de vida do projeto e sua gestão e a importância da gestão como competência essencial à engenharia civil. Os discentes conseguiram ver o BIM não apenas na perspectiva de ferramentas de modelagem 3D, mas como uma nova forma de se desenvolver projetos, de forma mais ampla que os sistemas CAD, ainda que tenham enfrentado algumas dificuldades com o aprendizado de novos tipos de softwares. A partir das discussões travadas ao longo do período, a maioria deles concluiu que a formação dos novos engenheiros civis deve obrigatoriamente contemplar o aprendizado do BIM e suas ferramentas e técnicas. Como limitação da experiencia foi apontado o fato de apenas duas horas semanais não serem suficientes para o aprendizado do BIM. Além disto, os alunos percebem os conflitos que surgem entre disciplinas como parte da dinâmica do desenvolvimento de projetos. Segundo os docentes, os alunos que cursam a disciplina eletiva de BIM alcançam um nível de proficiência básico, o que envolve a definição da geometria de pequenas edificações, contemplando alguns elementos básicos de modelagem MEP e 4D.

Ao longo da realização do projeto os alunos conseguem vivenciar o trabalho colaborativo e identificar conflitos. Neste sentido, conseguem desenvolver um nível de competência introdutório, referente ao primeiro estágio de adoção do BIM. Por outro lado, alunos que 
desenvolvem TCC, ao dispor de mais tempo, conseguem alcançar um nível de competência intermediário, conforme apresentado na Tabela 1.

\subsection{Dificuldades vivenciadas}

A modelagem desenvolvida a cada semestre coloca os discentes perante situações de conflito, com necessidade de negociação para alcance das soluções consensuais de projeto. Nesse sentido, a evolução da disciplina tem revelado algumas dificuldades não diretamente relacionadas com o BIM, mas com impacto direto no seu desenvolvimento. Apesar da totalidade dos discentes serem usuários habituais de computadores e tecnologias em geral, falta a eles familiaridade com questões básicas como conhecimento do sistema operacional ou organização e gestão de arquivos. Muito tempo é dispendido por conta de perda de arquivos de trabalho ou uso de versões desatualizadas. Além disso, o tempo dedicado ao aprendizado dos softwares é uma limitação para os objetivos da disciplina. Outra dificuldade é que, em geral, alunos periodizados têm poucas chances de cursar a disciplina, pela pressão do último semestre, quando precisam conciliar estágio, TCC e o encerramento do curso.

Os obstáculos enfrentados na oferta da disciplina são, em geral, condizentes com os relatos da literatura $[2,6,7]$, como a exigência de infraestrutura de laboratório com alta exigência de desempenho, além da visão disciplinar do currículo.

\subsection{Perspectivas}

Os alunos aprovados na disciplina podem propor projetos para serem desenvolvidos sob a orientação de algum dos professores responsáveis. Desses projetos já resultaram 15 TCC e outros tantos encontram-se em atualmente andamento, inclusive com parcerias com áreas como Estruturas e Materiais de Construção.

Atualmente, nas disciplinas sob responsabilidade do CCI (Desenho Técnico e Desenho Auxiliado por Computador) começa a ser feita uma apresentação do BIM e suas ferramentas no contexto de novas tecnologias, as quais prescindem do desenho como ferramenta de projeto. Considerando que as disciplinas com conteúdo de expressão gráfica totalizam 15 créditos, percebe-se que apenas uma reformulação na ênfase nos seus conteúdos possibilitaria a inclusão do BIM e suas tecnologias, sem necessidade de mudanças no currículo do curso.

Além disto, estão sendo estabelecidas parcerias com professores de outras disciplinas do curso de Engenharia Civil. A partir desses contatos, no $2^{\circ}$ semestre de 2018 o BIM começou a ser trabalhado em duas disciplinas da área de Estruturas: Bases para o dimensionamento de Estruturas e Estruturas Metálicas.

\section{Considerações finais}

A necessidade de implantação do BIM nos currículos de engenharia se torna premente e, neste sentido, pesquisadores e acadêmicos de instituições de ensino nacionais têm-se debruçado no estudo de estratégias para realizar tal ação. Como, de maneira geral, os currículos não dispõem de espaço para a inserção de novas disciplinas obrigatórias a opção tem sido a criação de disciplinas eletivas e grupos de pesquisa bem como a integração de disciplinas. A implantação do BIM no curso de Engenharia Civil da UFJF não escapa a esta regra. Desde o início da experiência, várias dificuldades têm sido vivenciadas e são semelhantes àquelas identificadas na literatura: a necessidade de investimentos em hardware e a ausência de métodos de ensino e materiais didáticos. Quanto aos discentes, faltam competências de TIC bem como tempo para aprendizado do BIM em função da carga horária do curso. Além disto, é notória a falta uma 
visão disciplinar do currículo que permita enxergar de forma mais abrangente a utilização do BIM no ciclo de vida de um projeto.

A partir da iniciativa de ensino de BIM em uma disciplina eletiva, o conceito começa a se disseminar no curso, motivando o desenvolvimento de um número crescente de TCC, o que possibilita alcançar um nível de competência intermediário.

Em outra direção, de maneira a fomentar o ensino de BIM, novos esforços têm sido realizados com a sua inserção em disciplinas existentes. Na disciplina de Expressão Gráfica são atualmente apresentadas as ferramentas BIM, permitindo um primeiro contato dos discentes com o conceito. Nas disciplinas "Bases para o Dimensionamento de Estruturas" e "Estruturas Metálicas", do núcleo profissionalizante, o conceito começa a ser trabalhado de maneira a fomentar a realização de projetos integrados de Arquitetura e Estruturas, em colaboração com a disciplina eletiva de BIM.

Do esforço empreendido até o momento vê-se que ainda existe um longo caminho para uma implantação efetiva do BIM no curso de Engenharia Civil da UFJF; ela deverá ocorrer de forma gradual, em diferentes etapas. Os primeiros passos já foram dados e os resultados são auspiciosos pois tem atraído o interesse dos discentes, fornecendo os subsídios necessários para começar a discutir as competências a serem desenvolvidas no currículo. Os autores deste trabalho acreditam o BIM não deve se restringir a uma disciplina ou mais disciplinas, mas permear todo o currículo, visão essa que requer de um trabalho com os docentes do curso para a derrubada de barreiras disciplinares e a consequente integração de conteúdos entre disciplinas. Uma grande contribuição é a ampliação da visão do discente quanto aos conflitos que surgem em um contexto de projeto, um assunto que comumente não é abordado em currículos de cursos de engenharia civil. Por outro lado, a disseminação do BIM no curso, mesmo que de forma ainda incipiente, tem despertado a atenção dos discentes, que passam a ser os indutores das mudanças a serem realizadas. Isto porque, ao vislumbrarem possibilidades de aplicação do BIM em outras disciplinas, eles acabam por despertar o interesse dos docentes pelo tema.

\section{Referências}

[1] BRASIL, "Decreto $\mathrm{n}^{\circ}$ 9.377, de 17 de maio de 2018. Institui a Estratégia Nacional de Disseminação do Building Information Modeling", accessed December 10, 2019, http://www.planalto.gov.br/ccivil_03/_ato2015-2018/2018/decreto/D9377.htm.

[2] K. Panuwatwanich, M. Wong, J. Doh, R.A. Stewart, and T.J. McCarthy, "Integrating Building Information Modelling (BIM) into Engineering Education: An Exploratory Study of Industry Perceptions using Social Network Data", in AAEE2013: Australasian Association for Engineering Education Conference (2013), Gold Coast, Queensland, Australia, pp. 1-9, accessed December 10, 2019, https://ro.uow.edu.au/eispapers/1920/.

[3] R. Sacks, C. Eastman, G. Lee, and P. Teicholz, BIM HANDBOOK: A guide to Building Information Modeling for owners, managers, designers, engineers, contractors, and Facility Managers. Hoboken, NJ: John Wiley \& Sons, 2018.

[4] R. Sacks, and R. Barak, "Teaching Building Information Modeling as an Integral Part of Freshman Year Civil Engineering Education", Journal of Professional Issues in Engineering Education and Practice, vol. 136, pp. 30-38, March 2009. doi: https://doi.org/10.1061/(ASCE)EI.1943-5541.0000003. 
[5] M.L. Andrade, and R. Ruschel, "Interoperabilidade de aplicativos BIM usados em Arquitetura por meio do formato IFC", Gestão \& Tecnologia de Projetos, vol. 4, pp.76111, November 2009. doi: https://doi.org/10.4237/gtp.v4i2.102.

[6] R. Sacks, and E. Pikas, "Building Information Modeling Education for Construction Engineering and Management: I, Industry requirements state of the art, and gap analysis", Journal of Construction Engineering and Management, vol. 139, November 2013. doi: https://doi.org/10.1061/(ASCE)CO.1943-7862.0000759.

[7] M.B. Barison, and Santos, E.T., "Tendências atuais para o ensino de BIM", in Encontro de Tecnologia de Informação e Comunicação na Construção (2011), Salvador-BA, Brasil, accessed December 15, 2019, http://www.uel.br/pessoal/barison/tese.htm.

[8] B. Becerik-Gerber, D.J. Gerber, and K. Ku, "The pace of technological innovation in architecture, engineering, and construction education: integrating recent trends into the curricula", Journal of Information Technology in Construction (ITcon), vol. 16, p. 411432, 2011. Accessed December 10, 2019, https://www.itcon.org/paper/2011/24.

[9] R. Ruschel C., M.L.V.X. Andrade, and M. Morais. "O ensino de BIM no Brasil: onde estamos?", Ambiente Construído, vol. 13, pp. 151-165, June 2013. ISSN 1678-8621.

[10]E.S. Checcucci, and A.L. Amorim, "Método para análise de componentes curriculares: identificando interfaces entre um curso de graduação e BIM". Pesquisa em Arquitetura e Construção, vol. 5, pp. 6-17, June 2014. doi: https://doi.org/10.20396/parc.v5i1.8634540.

[11] CBIC - Câmara Brasileira da Indústria da Construção, "Encontro Nacional sobre o Ensino de BIM impacta participantes com discussões e experiências compartilhadas", Brasília-DF, Brasil, 2018. Accessed December 10, 2019, https://cbic.org.br/encontro-nacional-sobre-oensino-de-bim-impacta-participantes-com-discussoes-e-experiencias-compartilhadas/.

[12] ANTAC - Associação Nacional de Tecnologia do Ambiente Construído, II Encontro Nacional sobre o Ensino de BIM(2019), Fortaleza-CE, Brasil. Accessed December 10, 2019, https://www.antaceventos.net.br/index.php/enebim2019/enebim/schedConf/.

[13] M.B. Barison, and E.T. Santos, "Ferramenta para planejamento de disciplina BIM", in Encontro Nacional de Tecnologia do Ambiente Construído (2014), Maceió-AL, Brasil, pp. 2933-2942. doi http://doi.org/10.17012/entac2014.489.

[14] L.S.R. Siqueira, "Aplicação das metodologias Building Information Modeling (BIM) e Aprendizagem Baseada em Problemas (ABP) no curso de graduação em engenharia civil / UFES: diagnóstico e recomendações”, 138f. Dissertação (Mestrado em Engenharia Civil), Faculdade de Engenharia, Universidade Federal do Espírito Santo, 2017.

[15] UFJF, "Projeto Político Pedagógico do curso de Engenharia Civil - 2017", accessed December 15, 2019, http://www.ufjf.br/engenhariacivil/files/2012/05/PPC-EngenhariaCivil-CCEC-16-Maio-2017-aprov-CONGRAD-Res.-59-2017-01-Jun.pdf.

[16] M. Aguilar, and W. Azevedo, "Formação em Engenharia Civil: Desafios para o Currículo na UFJF", in XLII Congresso Brasileiro de Educação em Engenharia (2014), Juiz de Fora, Minas Gerais, Brasil, accessed December 15, 2019, http://www.abenge.org.br/cobenge/arquivos/5/Artigos/129281.pdf.

[17]T.E. French, Desenho Técnico e Tecnologia Gráfica, $7^{\mathrm{a}}$ Ed. Atualizada, revisada e ampliada. São Paulo: Globo, 2005. 